[24] Gross, P. A., Neu, H. C., Aswapokee, P., van Antwerpen, C., and Aswapokee, N.: Death from nosocomial infection: experience in a university hospital and a community hospital. Am. J. Med. 68 (1980) 219-223.

[25] Celis, R., Torres, A., Gatell, J. M., et. al.: Nosocomial pneumonia: multivariate analysis of risk and prognosis. Chest 93 (1988) 318-324.

[26] Kappstein, I., Schulgen, G., Friedrich, T., et. al.: Incidence of pneumonia in mechanically ventilated patients treated with sucralfate or cemetidin as prophylaxis for stress bleeding bacterial colonization of the stomach. Am. J Med. 91 (suppl2A) (1991) 125S-131S.

[27] Hooton, T. M., Haley, R. W., Culver, D. H. White, J. W., Morgan, W. M., and Caroll, R.C.: The joint association of multiple risk factors with the occurrence of nosocomial infection. Am. J. Med. 70 (1981) 960-970.

[28] Cruse, P. J. E., and Foord, R.: The epidemiology of wound infection. A 10-year prospective study of 62,939 wounds. Surg. Clin. North Am. 60 (1980) 27-40.

[29] Mishriki, S. F., Law, D. J. W., and Jefferey P. J.: Factors affecting the incidence of postoperative wound infection. J. Hosp. Infect. 16 (1990) 223-230.

[30] Simchen, E., Stein, H., Sacks, T. G., Shapiro M., and Michel, J.: Multivariate analysis of determinants of postoperative wound infection in orthopaedic patients. J. Hosp. Infect. 5 (1984) 137-146.

[31] Jamulitrat, S., Mekanavin, U., and Thongpiyapoom, S.: Factors affecting mortality outcome and risk of developing nosocomial bloodstream infections. Infect. Control Hosp. Epidemiol. 15 (1994) 163-170.

Verfasser:

Prof. Dr. H. Rüden, Dr. P. Gastmeier, Dr. N. Wischnewski, Dr. G. Kampf, Institut für Hygiene der Freien Universität Berlin; Dr. T. Hauer, Prof. Dr. F. Daschner, Institut für Umweltmedizin und Krankenhaushygiene, Freiburg; Dr. J. Schlingmann und Prof. Dr. rer. nat. M. Schumacher, Institut für med. Binmetrie und Med. Informatik der Universität Freiburg

Korrespondenzanschrift:

Prof. Dr. Henning Rüden, Institut für Hygiene der Freien Universität Berlin, Nationales Referenzzentrum für Krankenhaushygiene, Hindenburg. damm 27, 12203 Berlin

\title{
Der Bundeslebensmittelschlüssel (BLS II.2)
}

\author{
Von L. I. Dehne, Ch. Klemm, G. Henseler, E. Hermann-Kunz und K. W. Bögl
}

\section{Zielsetzung des Bundeslebensmittelschlüssels (BLS)}

Der BLS besteht aus einem Lebensmittelcodiersystem (BLS-Schlüssel) und einer Nährstoffdatenbank und wurde als Standardinstrument für die Auswertung von Verzehrserhebungen entwickelt. Durch seine einheitliche Verwendung sollen Abweichungen bei der Auswertung von epidemiologischen Studien vermieden werden, die $z$. B. durch die Anwendung unterschiedlicher Nährwerttabellen, Bezugsgewichte, Nährstoffverlustmodelle oder die individuelle Festlegung fehlender Analysenwerte auftreten. Der BLS soll die Auswertung von epidemiologischen Studien erleichtern und eine Vergleichbarkeit der Ergebnisse verschiedener Verzehrstudien bzw. deren Fortschreibung ermöglichen.

\section{Konzeption und Aufbau des BLS}

Der BLS enthält 11878 Lebensmittel in verschiedenen Verarbeitungsstufen (z. B. roh, gegart, getrocknet, Konserve) und weist für jedes Lebensmittel bis zu 158 Lebensmittelinhaltsstoffe aus, z. B. die Gehalte an Fett, Fettsäuren, Protein,
Aminosäuren, Vitaminen, Mineralstoffen, Spurenelementen etc. Jedes Lebensmittel ist mit einer Schlüsselnummer und einem Textfeld in deutscher und englischer Sprache eindeutig charakterisiert.

Die Inhaltsstoffe der im BLS enthaltenen Lebensmittel setzen sich aus analytischen Werten (sogenannten Grundwerten) einerseits, und berechneten Werten andererseits zusammen. Werte werden dann berechnet, wenn auf keine oder keine validen Analysendaten zurückgegriffen werden kann. Auf dieser Basis ergibt sich folgende Datenstruktur: Von den insgesamt $11878 \mathrm{Da}$ tensätzen stellen 1265 sogenannte Grundlebensmittel dar, denen analytisch gewonnene Werte zugrunde liegen. Durch Verlustmodellrechnungen werden daraus die Daten für 1236 industriell hergestellte Lebensmittel und 1382 im Haushalt durch Garen zubereitete Lebensmittel berechnet. Für 2238 Lebensmittel werden die Werte mit Hilfe von Standardrezepturen ermittelt. Bei 760 Lebensmitteln werden die Werte ähnlicher Lebensmittel übernommen. Auf der Basis der vorgenannten Lebensmittel werden 3102 Gerichte und Menükomponenten zusammengestellt. Der BLS beinhaltet zudem 1148 Gruppenwerte. Die Bearbeitung des
BLS hat zu Umgruppierungen von Lebensmitteln geführt. Aus Kompatibilitätsgründen wurden bei diesen Lebensmitteln neben der Neuzuordnung auch der bisherige BLS-Code erhalten. Daraus resultiert eine Verdoppelung dieser Datensätze. Die insgesamt 747 Datensätze sind entsprechend gekennzeichnet und in einem neuen Update zur Streichung vorgesehen.

Diese Datenstruktur macht deutlich, daß die Nährwertdaten im BLS zur überwiegenden Anzahl mittels Algorithmen und Modellrechnungen generiert werden. Die daraus entstehenden Fehlermöglichkeiten und Ungenauigkeiten sind bei der Anwendung des BLS zur Auswertung von Verzehrserhebungen vertretbar, können jedoch bei der Anwendung des BLS zur Berechnung strenger Diäten von Bedeutung sein. Dieser Hinweis erfolgt vor dem Hintergrund, daß der BLS in zunehmendem Maße auch zur Berechnung von Kostplänen und Diäten verwendet wird und häufig in entsprechenden PC-Nährwertberechnungsprogrammen implementiert ist [1]. Insbesondere wenn solche Diäten die Meidung einzelner Minorbestandteile von Lebensmitteln, wie z.B. Phenylalanin zum Ziel haben, sollten entsprechende BLS-Angaben nicht ungeprüft übernommen werden. 


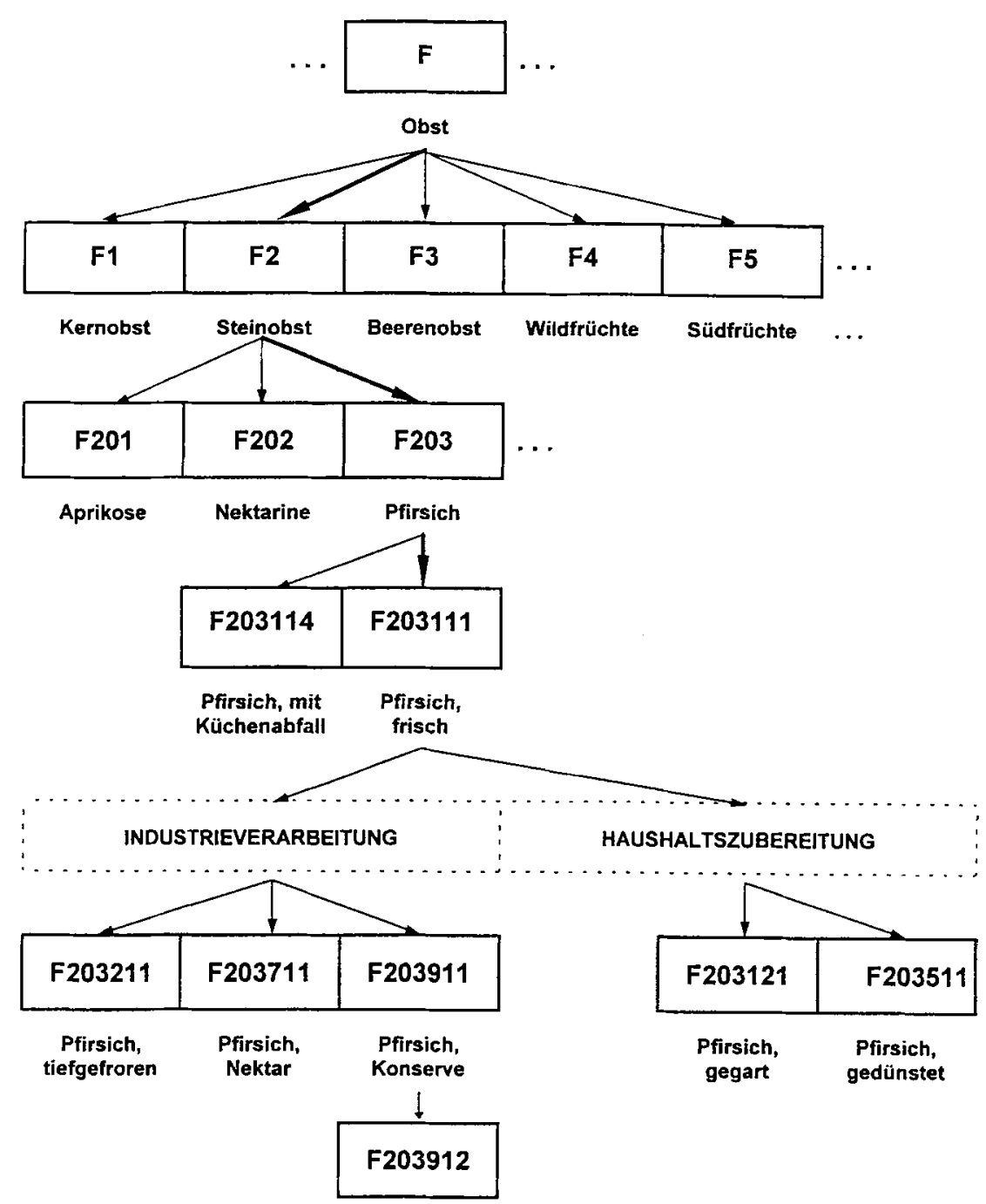

Pfirsich, Konserve abgetropft

Abbildung 1: Das BLS-Schlüsselsystem am Beispiel der Lebensmittelgruppe »F«.

\subsection{Das BLS-Schlüsselsystem}

Jedes Lebensmittel ist mit einer eindeutigen siebenstelligen Schlüsselnummer gekennzeichnet. Der Aufbau des Schlüssels ist in Abbildung 1 am Beispiel der Lebensmittelgruppe »Früchte» erläutert:

Die erste Stelle des Schlüssels, ein Buchstabe, beschreibt die Lebensmittelhauptgruppe, hier F für Früchte. Die zweite Stelle beschreibt die Lebensmitteluntergruppe, also z. B. Steinobst. Die dritte und vierte Stelle beschreiben das Einzellebensmittel, in diesem Fall Steinobstarten wie Aprikose oder Pfirsich. In der fünften Stelle ist die Industrieverarbeitung, z. B. Pfirsichkonserve, verschlüsselt. Die sechste Stelle gibt die Art stammen Nährwerttabellen, wissenschaftlichen Publikationen oder Mitteilungen der Bundesforschungsanstalten. Eine Validierung dieser Daten erfolgt in Zusammenarbeit mit dem Arbeitskreis der Beauftragten für LebensmittelNährstoffdaten im Geschäftsbereich des Bundesministeriums für Ernährung, Landwirtschaft und Forsten.

\section{Abgeleitete Analysenwerte}

Wie in Abbildung 1 am Beispiel von Früchten erläutert, werden im BLS ähnliche Lebensmittel in Gruppen zusammengefaßr. Fehlende Analysenwerte (missing values) eines Lebensmittels einer solchen Gruppe werden von einem ähnlichen Lebensmittel dieser Gruppe übernommen. Dies zeigt ein Beispiel aus der Gruppe der Fruchtgemüse: Fehlende Analysendaten für die Fleischtomate werden von der roten Tomate übernommen. Auf diese Weise entsteht auch für die weniger umfassend analysierte Fleischtomate ein kompletter $\mathrm{Da}$ tensatz.

Berechnung von Werten auf der Basis von Komponenten und Rezepturen

In vielen Fällen sind einzelne Lebensmittel chemisch-analytisch sehr umfassend charakterisiert worden, z. B. Magerfleisch von Rind und Schwein, Fettgewebe, Sehnen oder Mehl, Hefe, Butter und pflanzliche Fette. Weit weniger $\mathrm{Da}$ ten liegen jedoch für aus diesen $\mathrm{Be}$ standteilen hergestellte Erzeugnisse vor, z. B. für die große Vielzahl von Wurstwaren oder Brot und Backwaren. Im BLS wird die chemische Zusammensetzung solcher Erzeugnisse auf der Basis ihrer Einzelbestandteile und einer Standardrezeptur berechnet. Bei diesen Berechnungen werden gesetzliche Bestimmungen über die Beschaffenheit eines Lebensmittels und die Leitsätze des Deutschen Lebensmittelbuches berücksichtigt. In die Rezepturen gehen auch herstellungsbedingte Nährstoff verluste ein.

Auf diese Weise mittels sogenannter Mischungsrechnungen generierte Lebensmittel sind im BLS vor allem in den Gruppen B (Brot), D (Dauer- und Feinbackwaren) und W (Wurst- und Fleischwaren) zu finden.

\section{Indirekte Berechnung von Werten}

Während für die Hauptbestandteile Fett, Eiweiß, Kohlenhydrate und Ballaststoffe der Lebensmittel eine vergleichsweise große Anzahl an Analysen- 
daten zur Verfügung steht, sind Angaben über die Zusammensetzung dieser Bestandteile, also z. B. die Aminosäuren- oder Fettsäurenzusammensetzung, sehr lückenhaft. Diese Lücken werden im BLS auf der Grundlage der verfügbaren Literaturdaten durch eine indirekte Berechnung geschlossen. Bei dieser Art der Berechnung werden die einzelnen Bestandteile, z. B. die Fettsäuren, als Prozentzahl des Gesamtfettgehaltes eines Analysenwertes umgerechnet. Anschließend werden mit Hilfe der Prozentzahlen die Anteile der Fettsäuren auf den Fettgehalt des jeweiligen Lebensmittels im BLS umgelegt. Aufgrund der lückenhaften Datenlage erlaubt dieses Verfahren meist eine nur grobe Annäherung.

\section{Berechnung der Gruppenwerte}

Bei der Auswertung von Verzehrserhebungen stößt man nicht selten auf undifferenzierte Angaben der Befragten, man habe z. B. Fleisch, Gemüse und Obst gegessen. Um solche Angaben ver- werten zu können, müssen auch diese Lebensmittelhauptgruppen, und sinngemäß deren Untergruppen wie z. B. Schweinefleisch, Blattgemüse oder $\mathrm{Zi}$ trusfrüchte, mit einem vollständigen Inhaltsstoffdatensatz versehen sein. Ziel für eine nächste BLS-Version ist es, diese Gruppenwerte auf der Basis der Verzehrhäufigkeit als gewichteter Mittelwert aller Lebensmittel der Gruppe festzulegen. In der aktuellen Version wird noch nicht einheitlich verfahren. Zum Teil wird die Gruppenfunktion von einem Einzellebensmittel der Gruppe übernommen oder es werden ungewichtete Mittelwerte aus den Lebensmitteln der Gruppe gebildet.

\section{Die Rechenfelder des BLS}

In den sogenannten Rechenfeldern finden sich Größen wie z. B. Energie- und Wassergehalte, Broteinheiten, Vitaminäquivalente oder Nährstoffquotienten. Es handelt sich um Angaben, die nach definierten Formeln aus den Inhaltsstoffdaten errechnet werden.
Berechnung von Gewichts- und Näbrstoffuerlusten

Die Gewichts- bzw. Nährstoffverluste der Lebensmittel infolge industrieller Verarbeitung bzw. haushaltsmäßiger Zubereitung werden im BLS mit Hilfe von Verlustfaktoren berechnet. Diesen Verlustfaktoren liegen allgemeine Erkenntnisse über das Verhalten eines Lebensmittels bzw. Lebensmittelinhaltsstoffes, z. B. bei der Garung, zugrunde.

Für bestimmte Lebensmittel im BLS ist der Küchenabfall angegeben. Unter Küchenabfall wird im BLS eine wasserund nährstofffreie Trockensubstanz verstanden. Das bedeutet, daß die Nähr stoffkonzentration pro $100 \mathrm{~g}$ Lebensmittel bei steigendem Küchenabfallanteil sinkt.

\section{Berechnung von Menüs und Menü-} komponenten

Jedes im BLS enthaltene Lebensmittel kann Komponente in einem Gericht bzw. Menü sein. Die Komponenten

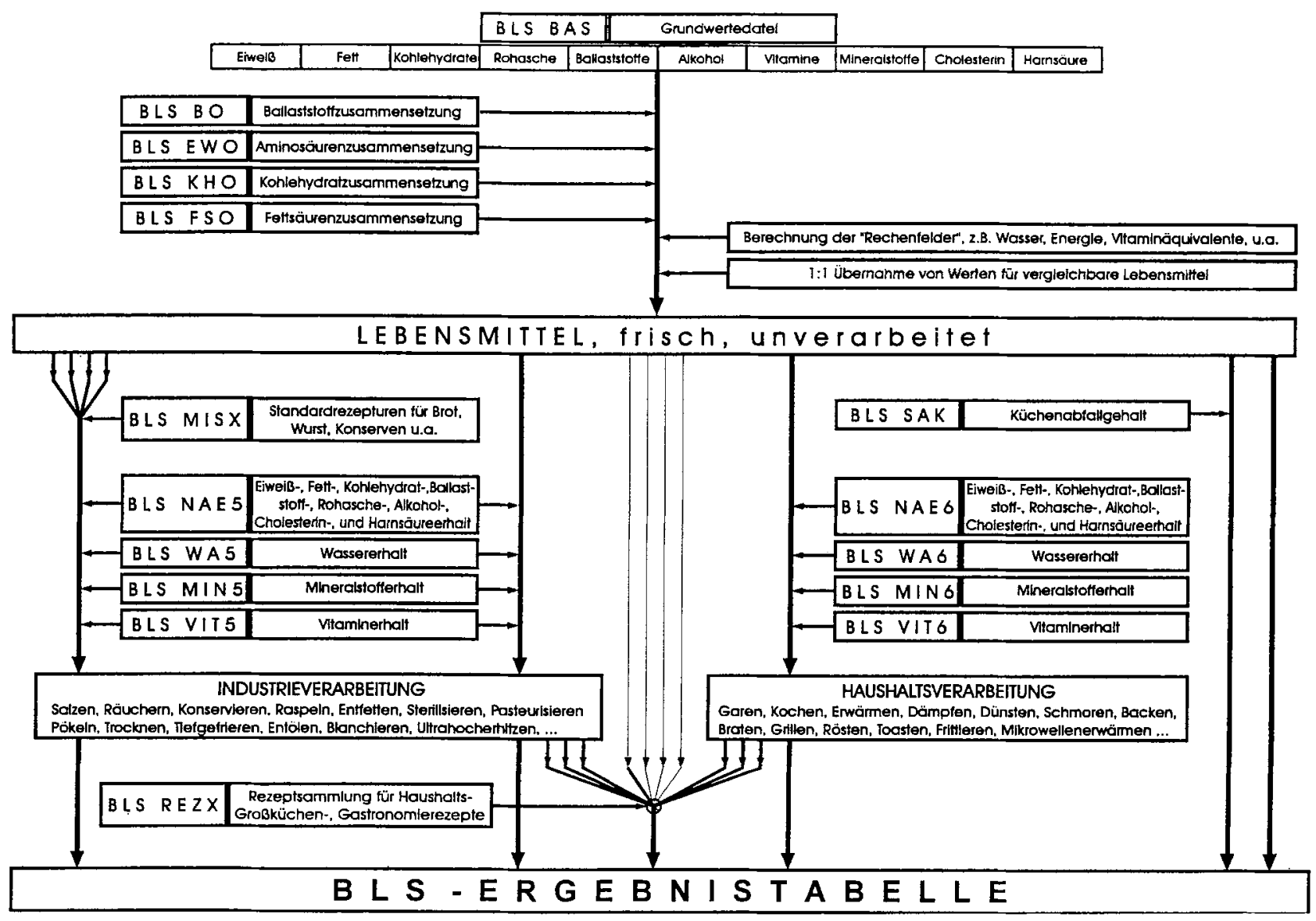

Abbildung 2: Die Entstehung der BLS-Ergebnistabelle (blsdat). 
können in roher, gegarter, gefrorener etc. Form oder mit/ohne Küchenabfall übernommen werden. In einer speziellen Rezeptdatei werden Art und Menge der Komponenten festgelegt, aus dem das Gericht bestehen soll. Es wird in Rezepte für Haushalt, Gastronomie und Großküche unterschieden. Eine Aufschlüsselung der Rezepte in ihre Komponenten ist dem BLS-Anwender z.Z. noch nicht möglich.

\subsection{Festlegung der Portionsgrößen}

Im aktuellen BLS ist zur Zeit eine " mittlere Portionsgröße« je Lebensmittel definiert. Die differenzierteren Portionsgrößen (wie Tasse, Glas, Kanne, Löffel, Teller etc.) befinden sich in Überarbeitung und werden in einer nächsten BLSVersion veröffentlicht werden.

\subsection{Das Berechnungsprogramm des BLS}

Die sogenannten Grundwerte sind der Ausgangspunkt für die Berechnung aller anderen Lebensmittel. Sie sind in der Datei mit der Bezeichnung BLS BAS eingetragen (Abb. 2).

In einem zweiten Schritt erfolgt die

Zuordnung der

Ballaststoffzusammensetzung

(BLS BO),

Aminosäurenzusammensetzung

(BLS EWO),

Kohlenhydratzusammensetzung

(BLS KHO) und die

Fettsäurenzusammensetzung

(BLS FSO) für diese Lebensmittel.

Anschließend erfolgt die Erstellung der Rechenfelder und die Zuordnung für die abgeleiteten Lebensmittel. Es liegen nun alle Grundwerte der Lebensmittel in der Form frisch/unverarbeitet vor.
Aus diesen werden im folgenden Programmschritt die Lebensmittel generiert, die auf der Basis von Einzelkomponenten und Standardrezepturen erstellt werden (BLS MISX).

Im nächsten Schritt erfolgt die Berechnung der Nährstoffveränderungen (Nährstofferhalt) bei der Industrieverarbeitung (BLS NAE5, BLS WA5, BLS MIN5, BLS VIT5) und bei der Haushaltszubereitung (BLS NAE6, BLS WA6, BLS MIN6, BLS VIT 6) sowie die Berechnung des Küchenabfalls (BLS SAK)

Den Abschluß bildet die Zusammenstellung der Rezepte und Rezeptkomponenten (BLS REZX), und es liegt nun die fertige BLS-Ergebnistabelle (BLS DAT) vor. Sie hat eine Größe von 11,2 Megabytes und ist im dBASE IV-Format oder ASCII-Format gegen eine Schutzgebühr erhältlich.

\section{Neuerungen im BLS II.2}

Einige Neuerungen bzw. Veränderungen im BLS II.2 gegenüber der Vorgängerversion lassen sich wie folgt zusammenfassen:

- Akrualisierung der Inhaltsstoffangaben für die Grundwerte. Dies führt u. a. zu einer $z$. T. erheblichen Reduzierung des Fettgehaltes von Fleisch und Fleischerzugnissen.

- Aufnahme der Inhaltstoffe $\alpha$-Tocopherol und nichtresorbierbare Oligosaccharide.

- Einführung eines Verlustmodells unter Berücksichtigung der Masseverluste für Fett, Wasser, Eiweiß etc.

- Neu aufgenommen wurden 998 Lebensmittel, wobei der Schwerpunkt auf den Bereichen Frischfleisch,
Frischfisch, Fischkonserven, Obstund Gemüsekonserven, Getränke, Rezeptzutaten und eirigen Gruppenwerten liegt.

- Es erfolgte eine bessere begriffliche Zuordnung der Menüs.

- Das BLS-Handbuch liegt jetzt auch in gedruckter Form vor [2].

\section{Ausblick}

Neben einer kontinuierlichen Datenpflege sollen für ein zum Jahreswechsel geplantes BLS-update schwerpunktmäßig die Rezepte und Rezeptkomponenten (Schlüssel $X$ und $Y$ ) überarbeiter werden. In diesem Zusammenhang soll es dem Anwender ermöglicht werden, die Rezepte in die Rezeptkomponenten aufzuschlüsseln und auch eigene Rezepte zu berechnen.

\section{Literatur:}

[1] Nährwertberechnung per PC. Computerprogramme und ihre Funktionen - Eine Übersicht. aid Special 9, 3334/1995.

[2] Der Bundesiebensmittelschlüsse] (BLS II.2) Konzeption, Aufbau und Dokumentation der Datenbank blsdat. BgVV-Hefte 2/1996.

\section{Anschriften der Verfasser:}

Dr. Lutz Ingo Dehne, Christine Klemm, Fachgebiet Allgmeine Lebensmitteltechnologie, Mikrowellen; Dr. Gernot Henseler, ZEBS; Dr. Klaus W. Bögl, Fachbereich Chemie und Technologie der Lebensmittel und Bedarfsgegenstände, Bundesinstitut für gesundheitlichen Verbraucherschutz und Veterinärmedizin (BgVV), Postfach 330013 , 14191 Berlin; Edelgard Hermann-Kunz, Fachgebiet Ernährungsbedingte Gesundheitsrisiken, Robert Koch-Institut, Postfach 6502 80, 13302 Berlin 